\title{
Optimization of Measuring Current for Chopper Low Resistance Comparator
}

\author{
R. M. Radetic ${ }^{1}$, D. R. Milivojevic ${ }^{2}$, V. M. Despotovic ${ }^{3}$ \\ ${ }^{1}$ Serbian Transmission Systems, Nade Dimic 40, 19210 Bor, Serbia, rradetic@ptt.rs \\ ${ }^{2}$ Mining and Metallurgy Institute, Zeleni Bulevar 35, 19210 Bor, Serbia, dragan.milivojevic@irmbor.co.rs \\ ${ }^{3}$ University of Belgrade, Technical Faculty of Bor, 19210 Bor, Serbia, vdespotovic@tf.bor.ac.rs
}

\begin{abstract}
The article describes systematic error analysis for realized chopper low-resistance comparator. The objective was to determine optimal working conditions, considering limited resolution (about $10 \mathrm{nV}$ ) and resistance variation due to self-heating caused by increase of measuring current. The analysis and performances of realized chopper low-resistance comparator prototype instrument confirmed the initial assumptions of applied principles. The error for $1 \mathrm{~A}$ measuring current is $1.15 \mathrm{ppm}$ in the measuring range of 10 $\mathrm{m} \Omega$. It is possible to use the same principle for calculation of optimal measuring current and measurement error in other measurement ranges, as well.
\end{abstract}

Keywords: Low-resistance measurement, chopper comparator, error analysis

\section{INTRODUCTION}

A $\mathrm{N}$ IMPROVEMENT of the chopper method for elimination of parasite voltages in the procedure of a small resistance comparison and measurement, and the design of prototype instrument are presented in [1] - [2]. Very high accuracy in resistance comparison and measurement is achieved $(0.08 \mathrm{ppm}$ for 1,000 independent measurement attempts and $0.26 \mathrm{ppm}$ for 100 attempts). The results of prototype instrument testing show the possibility of achieving $10 \mathrm{nV}$ voltage resolution by voltage measurement at the resistor terminals. The resistance measuring range is $10 \mathrm{~m} \Omega$. The applied method uses a current pulse with the amplitude 1 A, which for resistance in this measurement range gives maximal RI voltage of $10 \mathrm{mV}$. If the electric current intensity increases by measuring with constant resolution,, the relative error decreases due to higher voltage on the resistor terminals. On the other hand, the increasing of the measuring current produces greater power dissipation and higher resistor temperature. In a case when the temperature is changing, the resistance is changing as well [3]. This increases the error of measurement. These two opposite requirements have very significant influence on measurement quality. Certainly, the relationship between measuring current and relative error can be determined in order to provide optimal electric current intensity and minimal error [4]. The mathematical analysis of the described problem is given in the following chapter.

\section{SYSTEMATIC ERROR ANALYSIS}

Main sources of measurement errors in the chopperstabilized low resistance comparator prototype are parasite direct current (DC) and alternating current (AC) voltages. DC parasite voltages cause systematic error in measurement [5, 6]. On the other hand, AC parasite voltages cause dispersion of measured results around the mean value [1]. Further analysis is performed in order to determine optimal conditions for a minimal systematic error, as a consequence of two opposite requirements mentioned above.

\subsection{Systematic error due to limited resolution}

For the realized comparator, the value of measuring resistance $R_{\mathrm{X}}$ is:

$$
R_{X}=R_{R} \frac{U_{X}}{U_{R}}
$$

where $R_{\mathrm{R}}$ is standard (known) resistance, $U_{R}$ is voltage on $R_{R}$ terminals and $U_{X}$ is voltage on $R_{X}$ terminals

Measurement error due to limited resolution of voltage measurement $\Delta U_{\mathrm{X}}$ can be determined as:

$$
\Delta R_{X,(\Delta U)}=\frac{R_{R}}{U_{R}} \Delta U_{X}
$$

or as relative error:

$$
\delta_{U}=\frac{\Delta R_{X}}{R_{X}}=\frac{\Delta U_{X}}{U_{X}}
$$

\subsection{Systematic error due to power dissipation}

For the narrow temperature range the resistance variation depending on temperature can be expressed as:

$$
\mathrm{R}_{\mathrm{X}}=\mathrm{R}_{\mathrm{X} 0}(1+\alpha \Delta \vartheta)
$$

where $R_{X 0}$ is the resistance at the reference temperature $\vartheta_{0}, \alpha$ is the temperature coefficient of resistance and $\Delta \vartheta$ is the increase in temperature.

At low power dissipation, the resistor temperature change is proportional to power and can be expressed as:

$$
\Delta \vartheta=\mathrm{kP}
$$

where $k$ is coefficient ratio $(K / W)$. Now, the relationship between resistance variation and power can be shown as:

$$
\mathrm{R}_{\mathrm{X}}=\mathrm{R}_{\mathrm{X} 0}(1+\alpha \mathrm{kP})=\mathrm{R}_{\mathrm{X} 0}\left(1+\delta_{\mathrm{P}}\right)
$$


where $\delta_{\mathrm{p}}$ is resistance error due to power dissipation (self heating).

\subsection{Total measurement error}

In the worst case, the total measurement error can be determined from equations (3) and (6) as:

$$
\delta=\delta_{\mathrm{U}}+\delta_{\mathrm{P}}=\frac{\Delta \mathrm{U}_{\mathrm{X}}}{\mathrm{U}_{\mathrm{X}}}+\alpha \mathrm{kP}
$$

The realized chopper-stabilized low resistance comparator prototype uses the switched measuring current principle [7]. The controller switches the measuring current on and off and controls the functions of the voltage circuit analog switch. It is adjusted so that the duration of current pulse with the amplitude $1 \mathrm{~A}$ is $60 \%$ of one controller cycle [1]. During the remaining $40 \%$ of a cycle the current is switched off. Power dissipation in the resistor can be then determined as:

$$
P=\frac{t_{I}}{t_{I}+t_{P}} R_{X} I^{2}=a R_{X} I^{2}
$$

where $I$ is the electrical current pulse intensity, $t_{I}$ is the duration of current pulse (switched on), $t_{P} \mathrm{~s}$ the duration of current pulse (switched off) and $a$ is the duty cycle (for our comparator, $a=0,6)$. Now the total measuring error can be expressed as:

$$
\delta=\delta_{\mathrm{U}}+\delta_{\mathrm{P}}=\frac{\Delta \mathrm{U}_{\mathrm{X}}}{\mathrm{R}_{\mathrm{X}} \mathrm{I}}+\alpha \mathrm{kaR_{ \textrm {X } }} \mathrm{I}^{2}
$$

This mathematical expression gives the relation between total error and measuring current and it is illustrated in Fig.1. The figure shows the other two error components $\left(\delta_{U}\right.$ and $\left.\delta_{\mathrm{P}}\right)$, as well.

Minimum value of total measurement error can be determined by finding the first derivative and equalizing with zero:

$$
\frac{\mathrm{d} \delta}{\mathrm{dI}}=-\frac{\Delta \mathrm{U}_{\mathrm{X}}}{\mathrm{R}_{\mathrm{X}} \mathrm{I}^{2}}+2 \alpha \mathrm{kaR_{ \textrm {X } }} \mathrm{I}=0
$$

The solution gives the optimal measuring current in the form:

$$
\mathrm{I}_{\mathrm{OPT}}=\sqrt[3]{\frac{\Delta \mathrm{U}_{\mathrm{X}}}{2 \alpha \mathrm{kaR}_{\mathrm{X}}^{2}}}
$$

The minimal total measurement error can be determined as:

$$
\delta_{\mathrm{MIN}}=\frac{3}{2} \frac{\Delta \mathrm{U}_{\mathrm{X}}}{\mathrm{R}_{\mathrm{X}} \mathrm{I}_{\mathrm{OPT}}}
$$

The last expression shows that the use of the optimal measuring current will result in the error due to limited resolution twice greater compared to the error due to resistor self-heating. If we use equation (11) in (12), the minimal measurement error becomes:

$$
\delta_{\text {MIN }}=\frac{3}{2} \sqrt[3]{\frac{2 \alpha \mathrm{ka} \Delta \mathrm{U}_{\mathrm{X}}^{2}}{\mathrm{R}_{\mathrm{X}}}}
$$

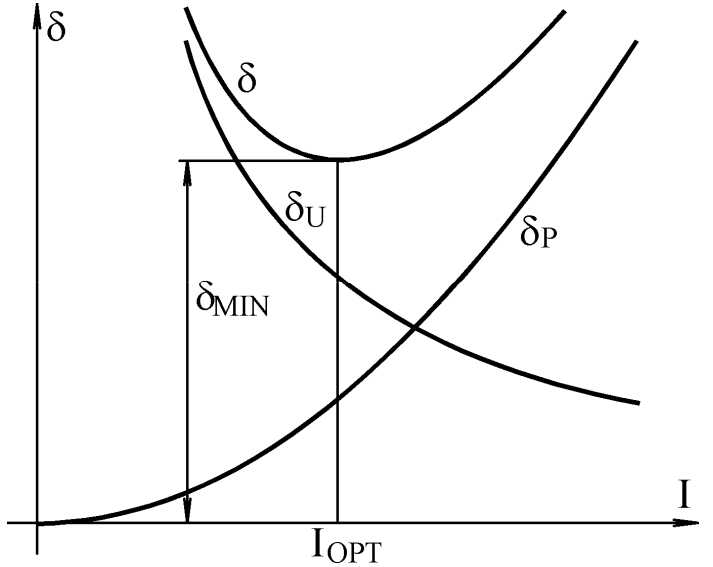

Fig.1 The error dependence on measuring current

\section{Practical Determination Of Optimal Current And TOTAL MEASUREMENT ERROR}

For the realized prototype instrument following parameters are assumed: $\alpha=10^{-5} 1 / K$ (the worst case), $k=2,5 \mathrm{~K} / \mathrm{W}$ (for Thompson type of resistors), $a=0,6$ (projected current pulse timing or duty cycle) and measuring resolution $\Delta U_{\mathrm{X}}=10 \mathrm{nV}$. Let us now use this values in equation (11) for the measuring resistance range of $R_{X M A X}=0,01 \Omega$. The optimal measuring current is now:

$$
\mathrm{I}_{\mathrm{OPT}}=\sqrt[3]{\frac{\Delta \mathrm{U}_{\mathrm{X}}}{2 \alpha \mathrm{kR}_{\mathrm{X}}^{2}}}=1,49 \mathrm{~A}
$$

The minimal total measurement error is then:

$$
\delta_{\mathrm{MIN}}=\frac{3}{2} \frac{\Delta \mathrm{U}_{\mathrm{X}}}{\mathrm{R}_{\mathrm{X}} \mathrm{I}_{\mathrm{OPT}}}=10^{-6}=1 \mathrm{ppm}
$$

Since the measuring current in the realized prototype comparator is fixed and has the amplitude $1 \mathrm{~A}$, the total measurement error in practical realization is:

$$
\delta=\frac{\Delta \mathrm{U}_{\mathrm{X}}}{\mathrm{R}_{\mathrm{X}} \mathrm{I}}+\alpha \mathrm{kaR_{ \textrm {X } }} \mathrm{I}^{2}=1,15 \cdot 10^{-6}=1,15 \mathrm{ppm}
$$

The increase of measurement error value due to deviation of current intensity is $0.15 \mathrm{ppm}$. Knowing that the realization of the current generator for integer values of current intensity is significantly simpler, such small differencies in accuracy of measurement can be considered as acceptable.

If we use equation (13) with parameters in practical realization, we can give common mathematical expression for our chopper stabilized low resistance comparator measurement error.

$$
\delta_{\text {MIN }}=\frac{0,22 \cdot 10^{-6}}{\sqrt[3]{R_{X}}}
$$

The last expression is very useful for calculation of total systematic measurement error for realized chopper stabilized low resistance comparator. The value of resistance $R_{X}$ defines the instrument measuring range. Theoretical results for measurement ranges 0.001-10 [ $\Omega$ ] are presented in Tab.1 [6]. 
Tab.1 Theoretical results for different measurement ranges

\begin{tabular}{|c|r|r|r|}
\hline $\begin{array}{c}\text { Measurement } \\
\text { range }(\Omega)\end{array}$ & $I_{\text {opt }}(A)$ & $P(m W)$ & $\begin{array}{c}\delta_{\text {min }} \\
(\mathrm{ppm})\end{array}$ \\
\hline \hline 0,001 & 6,93 & 29 & 2,2 \\
\hline 0,01 & 1,49 & 13 & 1,0 \\
\hline 0,1 & 0,32 & 6 & 0,47 \\
\hline 1 & 0,069 & 2,8 & 0,22 \\
\hline 10 & 0,149 & 1,3 & 0,1 \\
\hline & & & \\
\hline
\end{tabular}

\section{CONCLUSION}

Chopper-stabilized low resistance comparator prototype has been designed for very high accuracy in resistance comparison and measurement $(0.08 \mathrm{ppm}$ for 1,000 independent measurement attempts and $0.26 \mathrm{ppm}$ for 100 attempts) [1], [7], [8], Fig.2. The analysis of measurement error is carried out in order to define optimal values of measuring current and measurement error. The obtained results show very good coincidence of theoretical and experimental results.

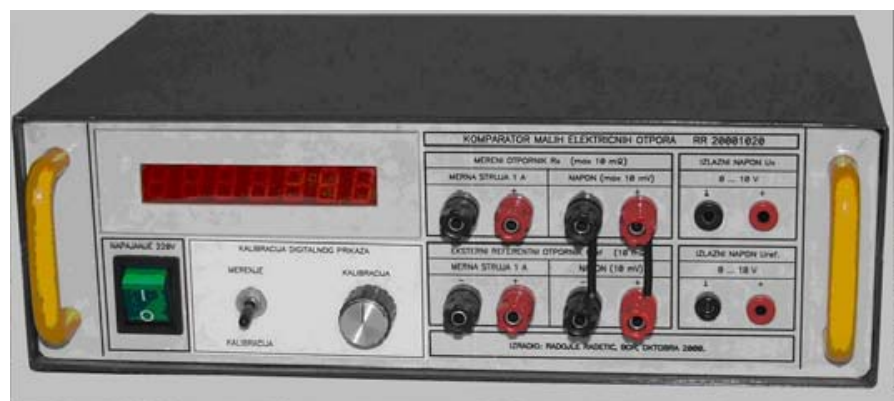

Fig.2 The realized low resistance comparator
Future activities will be focused on realization of measurement in different measurement ranges with the possibility of auto calibration. The direct connection to the subordinated PC is also a very useful activity. It could provide easy calculations, very illustrative result presentation and report collection.

\section{REFERENCES}

[1] Radetic, R.M., Milivojevic, D.R. (2009). Chopper stabilized low resistance comparator. Sensors, 9 (4), 2491-2497.

[2] Radetic, R., Zupunski, I. (2000). Low resistance comparator. In Proceedings of the Congress of Yugoslav Metrologists. Novi Sad, Serbia.

[3] Barney, G.C. (1988). Intelligent Instrumentation. Prentice Hall International.

[4] Boyes, W. (2003). Instrumentation Reference Book ( $3^{\text {rd }}$ ed.). Butterworth-Heinemann Publishing.

[5] Laughton, M.A., Warne, D.J. (Eds.) (2003). Electrical Engineer's Reference Book (16 ${ }^{\text {th }}$ ed.). Newnes- Elsevier Science.

[6] Schwertner, T. (2008) Obtaining More Accurate Resistance Measurements Using the 6-Wire Measurement Technique. White Papers. Keithley Instruments.

[7] Radetic, R., Zupunski I. (2003). Low resistance comparator test results. In Proceedings of the Congress of Yugoslav Metrologists. Belgrade, Serbia.

[8] Radetic, R. (2009). Low Resistance Electronic Comparator. $\mathrm{PhD}$ thesis, University of Novi Sad, Faculty of Technical Sciences, Novi Sad, Serbia.

Received November 23, 2009. Accepted February 2, 2010. 